\section{IN BRIEF}

\section{$\Rightarrow$ VIRAL INFECTION}

\section{The STING behind dengue virus infection}

Stabell et al. have revealed why human dengue viruses do not replicate to high titres in primate models. They found that the stimulator of interferon genes (STING) protein, which induces the production of type I interferon in infected cells to reduce viral titres, is cleaved by the dengue virus protease NS2B3 in humans but not in key primate models. STING cleavage occurred at an RG motif at amino acids 78 and 79, decreasing markers of an innate immune response and increasing viral replication in human cells. The analyses of STING sequences from all placental animals in Genbank, as well as from 16 non-human primate cell lines, revealed that only STING from three small apes and three small rodents encodes this RG motif. As introducing this RG motif into STING from rhesus macaque, marmoset and mouse rendered it susceptible to cleavage by dengue virus NS2B3, engineering model organisms so that their STING contains this motif could enhance the study of dengue viruses in animals.

ORIGINAL ARTICLE Stabell, A. C. et al. Dengue viruses cleave STING in humans but not in nonhuman primates, their presumed natural reservoir. eLife 2018, e31919 (2018)

\section{$\Rightarrow$ FUNGAL BIOLOGY}

\section{Finding IRESs in mycoviruses}

Cap-independent translation from internal ribosomal entry sites (IRESs) is initiated from viral mRNAs in animals and plants, but, until recently, no IRESs were known in fungal viruses. Chiba et al. have developed a dual-luciferase construct in which potential IRES sequences are inserted between codon-optimized Renilla (ORluc) and firefly (OFluc) luciferase; active IRES sequences are indicated by OFluc, and ORluc is an internal control. Using this system, the authors revealed that in the filamentous fungi Cryphonectria parasitica, sequences from the 5' UTRs of hypoviruses (namely Cryphonectria hypovirus), totiviruses and chrysoviruses - but not of mycoreoviruses, quadriviruses or partitiviruses - have IRES activity. IRES activities unveiled using this transformation-based assay were confirmed using an RNA transfection assay, validating this new experimental system. ORIGINAL ARTICLE Chiba, S., Jamal, A. \& Suzukia, N. First evidence for internal ribosomal entry sites in diverse fungal virus genomes. $\mathrm{mBio}$ 9, e02350-17 (2018).

\section{$\Rightarrow$ BACTERIAL PHYSIOLOGY}

\section{Nanowires under the microscope}

Shewanella oneidensis produce outer membrane extensions that are thought to function as nanowires that transport respiratory electrons to electron acceptors outside the cell. Subramanian et al. have developed a system in which S. oneidensis MR-1 forms outer membrane extensions on an electron microscopy grid inside a perfusion flow imaging platform. Monitoring the same extensions in this chamber with live fluorescence light microscopy followed by electron cryotomography revealed that these are chains of interconnected outer membrane vesicles with electron-dense particles on the interior and exterior. Comparing wild-type S. oneidensis MR-1 with S. oneidensis MR-1 null for cytochrome genes revealed that the electron-dense particles in wild-type S. oneidensis MR-1 are cytochromes. Based on the distance between cytochromes, the authors proposed that in these nanowires, electrons are transported by hopping between, and by the physical diffusion of, cytochromes.

ORIGINAL ARTICLE Subramanian, P. et al. Ultrastructure of Shewanella oneidensis MR-1 nanowires revealed by electron cryotomography. Proc. Natl Acad. Sci. USA https:// doi.org/10.1073/pnas.1718810115 (2018)
The ascomycete fungus Magnaporthe oryzae causes rice blast, a serious disease of cultivated rice that affects the global rice production. Infection is initiated when the fungus differentiates to form appressoria, which are specialized infectious structures, and penetrates the epidermal layer of the plant leaf using a narrow extension known as a penetration peg. Plant tissue invasion occurs via hyphae and the fungus spreads to neighbouring cells often through pit fields, which contain plasmodesmata (which are channels that traverse the cell walls of plant cells). However, the molecular mechanisms of cell-to-cell spread of the fungus and its ability to evade plant defences and establish colonization are not well understood. In this study, Sakulkoo et al. investigated $M$. oryzae colonization and spread and found that the mitogen-activated protein kinase (MAPK) pathway has a central role in plant cell invasion.

Using transmission electron microscopy analysis of rice sheath cells infected with a pathogenic $M$. oryzae strain, the authors confirmed the previously observed constriction of invasive hyphae as they cross the plant cell wall. Moreover, callose deposition (a plant defence mechanism that leads to closure of intercellular plasmodesmata) was not observed at early stages of infection (that is, 27 hours post-inoculation), which suggests that $M$. oryzae inhibits the deposition of plasmodesmal occlusion materials. To investigate the molecular mechanisms that control invasive growth, the authors used a chemical genetic approach to inactivate the fungal MAPK

\title{
VIRAL EVOLUTION
}

\section{Treading the same path}

RNA viruses cause some of the most notorious human diseases, from influenza to haemorrhagic fevers. Our understanding of the evolutionary histories of these viruses is largely limited to those found in mammals and birds, as they are thought to be the natural reservoir for many viruses that cause disease in humans. Yet, RNA viruses have been hypothesized to have an ancient origin. To gain a better understanding of their long-term evolution, genomic surveys of RNA viruses that are associated with diverse hosts are required. Now, a study reports the discovery of 214 RNA viruses from diverse vertebrates that reveal new insights into host-virus relationships across evolutionary timescales.

To better understand the origin and evolutionary history of vertebrate viruses, Shi et al. performed a large-scale meta-transcriptomics survey of potential RNA viruses in 186 vertebrate species that represent the vast diversity of the phylum Chordata, including mammals, reptiles, birds, amphibians and different classes of fish. The authors extracted total RNA from the gut, liver and lung or gill from these animals and performed high-throughput RNA sequencing. In their analysis of the sequences, they screened for RNA viruses and identified 214 new putative viral species of which 196 can be considered vertebrate-specific. The authors observed that every vertebrate-specific viral family or genus that is known to infect mammals and birds is also present in reptiles, amphibians or fish, and, moreover, they found a number of viruses that belong to families that had not been previously found in fish or amphibians. For example, they identified influenza viruses in jawless fish, amphibians and ray-finned fish, the latter of which was found to form a sister-group to human influenza $B$ virus. They also found that the newly described viruses shared 